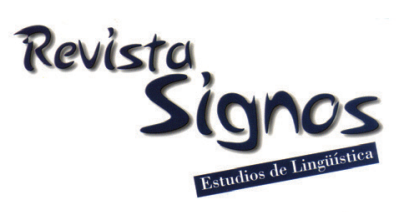

\title{
El sujeto y la subjetividad: Variación del pronombre ‘yo' en géneros conversacionales y de los medios de comunicación del español de Canarias ${ }^{1}$
}

The subject and the subjectivity: Variation of 'yo' in conversational
and mass-media genres of Canarian Spanish

María José Serrano

UNiversidad DE LA LAGUNA

EsPaña

mjserran@ull.edu.es

Recibido: 21-VI-2012 / Aceptado: 7-VIII-2013

\section{Resumen}

Tradicionalmente, los textos de naturaleza oral han sido considerados como los que muestran el mayor nivel de subjetividad y egocentrismo lingüístico. A partir de la prominencia cognitiva y de la informatividad textual como propiedades de la variación entre la expresión y la omisión del pronombre de sujeto 'yo' en un conjunto de géneros de los medios de comunicación y conversacionales del Corpus Conversacional del Español de Canarias, se analiza la dimensión de la subjetividad lingüística, entendida como la inscripción del referente en el contenido comunicativo que emite, de acuerdo a las características interactivas de cada género textual. Aquellos que involucran en mayor medida al hablante mediante el uso del pronombre 'yo' expreso preverbal son los que adquieren más subjetividad, tales como los informativos-debates. Esto podría indicar que algunos textos de los medios de comunicación son más subjetivos que los propiamente conversacionales.

Palabras Clave: Variación, sujeto, subjetividad, discurso, estilo comunicativo. 


\begin{abstract}
Conversational texts have usually been considered subjective and egocentric. From notions like cognitive salience and textual informativity which characterize expression and omission of the subject pronoun yo, subjectivity will be analyzed in some media and conversational genres of Canarias' spoken Spanish along with interaction properties of each text. Those which involve a speaker through the use of this pronoun in preverbal position should adopt subjectivity, like in news-debates genre. This seems to indicate that some media genres are more subjective than conversational ones.
\end{abstract}

Key Words: Variation, subject, subjectivity, discourse, communicative style.

\title{
INTRODUCCIÓN
}

Las formas de primera y segunda persona del paradigma pronominal en español están consideradas como los pronombres intrínsecamente personales, y esto es debido a que los referentes suelen formar parte de la interacción por estar presentes de algún modo en el contexto discursivo. 'Yo', 'tú', 'usted' y sus plurales se construyen mutuamente en la interacción; la segunda persona es el resultado de su enunciación por parte de la primera. Al mismo tiempo son reversibles; a partir del intercambio comunicativo 'yo' se transforma en 'tú' y viceversa, y de alguna manera se oponen (Rosengren, 1974). El alto grado de animacidad o concreción de los referentes de los sujetos pronominales es otra de las características de 'yo/nosotros', 'tú/vosotros', 'usted/ustedes', pero disminuye, está ausente o es menos relevante en 'él/ella' ya que los referentes de tercera persona pueden ser animados o no (Enríquez, 1984; Langacker, $2000)^{2}$. En cambio, esta última no solo no está presente en el contexto discursivo, sino que se constituye a partir de las dos primeras, que formulan algo sobre alguien o algo. Todo ello ha hecho dudar sobre su auténtica naturaleza de 'persona' (Benveniste, 1977).

Se ha considerado que los pronombres personales son una indicación de la subjetividad en la lengua, ya que implican, en mayor o menor medida y según la persona de que se trate, al hablante (Benveniste, 1977). En este sentido, la primera persona del singular 'yo', referida a la persona que habla en un discurso e interacción concreta, constituiría la máxima expresión de dicha subjetividad, entendida como implicación de la individualidad del hablante en su contraposición con otras personas del paradigma (Rosengren, 1974). Por lo tanto, la primera y la segunda persona se ordenan no solamente en cuanto a la referencia, animacidad o concreción de sus referentes, sino también en cuanto a la mayor implicación o perspectiva del hablante sobre el enunciado; la referencia a la propia persona constituye la más evidente representación de la perspectiva del ego y de la subjetivización, que se diferencia claramente de la segunda, y esto se produce en mayor medida con las formas del singular. Benveniste (1977) considera que 'yo' y 'tú' constituyen una doble correlación: por una parte, una de personalidad donde 'yo' se opone a 'tú', y otra de subjetividad: 
el primero es interior o inherente al enunciado, mientras que el segundo es exterior o externo al mismo. Cuando se sale del 'yo', se encuentra el 'tú', que es la persona imaginable más allá del 'yo'.

De forma general, esto responde a la distinción que se produce en todos los órdenes del conocimiento entre la esfera interna de los sujetos humanos y la externa de las terceras personas y los objetos.

La tradición gramatical ha destacado que la actitud del sujeto hablante frente a los hechos es lo que propicia el uso de este pronombre (Hale, 1966; Ejarque, 1977; Enríquez, 1984; Fernández Ramírez, 1987) y esta idea, aunque permanece sin desarrollar en relación a la subjetividad, resulta especialmente útil en lo que respecta a la variación entre la expresión y la omisión de 'yo'.

En este trabajo se tratará de dilucidar la relación existente entre la frecuencia en el uso de la primera persona del singular (yo), los géneros textuales en los que aparece y la dimensión cognitiva de la subjetividad. Está estructurado del siguiente modo; en primer lugar se determinará el marco teórico y conceptual sobre el que se sustenta este estudio (apartado 1) y la metodología empleada (apartado 2). A continuación analizaremos las propiedades cognitivas que fundamentan la variación entre la expresión (preverbal o posverbal) y la omisión de este pronombre en distintos géneros de los medios de comunicación y conversacionales (apartado 3). Una vez establecido el significado discursivo-cognitivo de cada variante y su frecuencia en cada tipo de texto, observaremos el continuum estilístico que crean las variantes basado en el polo de la objetividad-subjetividad lingüísticas, que constituyen los resultados (apartado 4).

\section{Marco teórico y conceptual}

Los avances más recientes en el estudio de la variación lingüística, y particularmente de la que se da en el plano sintáctico, permiten superar la habitual visión de las variantes como meras alternativas para comunicar un mismo contenido, que se limitan a covariar con los caracteres sociales de los hablantes o con el nivel de formalidad de la situación. Hoy es posible sostener que las formas gramaticales en alternancia siempre comportan diferencias comunicativas, fundamentadas en valores pragmático-discursivos y cognitivos, y que los hablantes las utilizan en la interacción como recursos de estilo, esto es, como formas de construir significados. La variación en el plano morfosintáctico y su relación con el estilo comunicativo no son ideas nuevas: forman parte de las teorías sugeridas por Lavandera (1984) desde los primeros tiempos del variacionismo laboviano, como consecuencia de la aplicación de los procedimientos del plano fonético-fonológico a la morfosintaxis.

Partiendo de esta base teórica, el uso de cualquier pronombre de sujeto viene determinado por la experiencia del hablante en relación con su entorno, sus necesidades comunicativas y las características concretas de la interacción. Sin embargo, no hay que perder de vista que la lengua española posibilita la omisión del 
sujeto, de modo que esta forma adopta un significado diferente cuando se expresa y cuando no lo hace, lo cual influye notablemente en la percepción de su referente en la escena comunicativa y en la dimensión de la subjetividad que tradicionalmente se le ha adjudicado (Benveniste, 1977). Para ello, un enfoque cognitivo como el que adoptamos resulta fundamental para entender esta variación.

Estudiaremos esta variación en dos grandes tipos de géneros textuales: los conversacionales y los de los medios de comunicación, según se detalla en el apartado de la metodología. El género textual es una noción sociocultural que puede ser muy útil para caracterizar estilos comunicativos, ya que en ellos se desarrolla la actividad comunicativa humana. Sin embargo, no es fácil formular una definición del género textual, ni diferenciarlo de otras categorías análogas como 'texto', 'tipo de discurso' o 'registro' (Biber \& Conrad, 2009). En este trabajo utilizaremos el término 'género' como los textos cuyas características pueden ser definidas por el uso de ciertos patrones lingüísticos que se producen en ciertas situaciones comunicativas, y que pueden estar socialmente institucionalizados, tales como artículo periodístico, debate político, magazine o programa de entretenimiento televisivo, etc. Por su parte, como indicamos más abajo, las conversaciones espontáneas pueden considerarse como un 'protogénero', de donde deriva el resto de las actividades discursivas. Dado que ofrecen características textuales muy diferenciadas, en este trabajo analizaremos los textos conversacionales y los de los medios como dos grandes grupos de géneros diferenciados.

Uno de los aspectos determinantes para entender la variación entre las distintas variantes del sujeto pronominal 'yo' es el tipo de texto donde aparece; de forma general, este pronombre se ha estudiado como una forma muy frecuente en la conversación espontánea o coloquial, donde, como señala Padilla García (2001), el hablante necesita dejar huella de su presencia a partir de la necesidad de reintroducción y de mantenimiento de la atención del interlocutor. De forma general, se considera que el uso de los sujetos, y especialmente 'yo', es característico y casi prototípico de los textos conversacionales o 'coloquiales', mientras que en otros géneros con aparente menor intensidad de interacción, este decrece. Sin embargo, estas generalizaciones no han sido suficientemente contrastadas, en buena medida porque no se han enfocado como posibilidades variables que aparecen en determinados géneros textuales (Dorgeloh \& Wanner, 2010) y porque se ha utilizado una idea demasiado vaga de la subjetividad lingüística. Una de las acepciones más recurrentes de la subjetividad es la que alude a la 'connotación', como oposición a la objetividad, que sería 'denotativa', y es la que con mayor frecuencia ha caracterizado de forma constante a la lengua oral y al denominado 'lenguaje coloquial', y es la que predomina, al menos, en los estudios de lingüística hispánica (Criado de Val, 1973; Narbona, 1991; Vigara Tauste, 2005; Hernando Cuadrado; 1988, entre otros). A esta caracterización se añade, normalmente, la de la pobreza, simplicidad, desorden y redundancia del denominado 'lenguaje coloquial' (Hidalgo Navarro \& Pérez Giménez, 2004). 
De hecho, el concepto de subjetividad aplicado a la lingüística general tiene diversas vertientes teóricas, y no precisamente uniformes; bajo esta denominación suelen aparecer dimensiones teóricas de distinto signo, tales como 'no-objetividad', 'imprecisión', 'ambigüedad', 'vaguedad', 'incertidumbre' y 'medidas no objetivas'3 (Chen, 2008). Aunque no explícitamente, todas estas nociones, de una forma u otra, han pretendido caracterizar la subjetividad, bien como concepto antropocéntrico que permite la percepción de la realidad a través de las distintas lenguas (Wierzbicka, 1988), bien como una cuestión epistemológica que se refiere a la condición humana y su identidad (Atkins, 2005). Es evidente que el componente humano está presente en la idea de subjetividad, y que este debería tener repercusiones sobre la estructura de las lenguas, pero es necesario precisarlo y observarlo en interacciones y construcciones lingüísticas concretas. Finegan (1995: 1) define la subjetividad como "el punto de vista personal del hablante o la huella que deja en el habla", si bien no lo asocia a funciones gramaticales concretas. Por su parte, Kerbrat-Orecchioni (1980) enfoca la subjetividad desde una perspectiva textual, asociándola a la gama de las distintas modalidades enunciativas del hablante (Calsamiglia \& Tusón, 2007). En una dimensión más amplia, Englebretson (2007) enmarca la subjetividad como una de las consecuencias (aunque no la única) de la postura que el hablante adopta en sus enunciados, que se manifiesta a través del uso de ciertas construcciones lingüísticas.

Al lado de las vagas y diversas dimensiones que apunta Chen (2008), la subjetividad lingüística se ha relacionado también con la noción de 'egocentrismo'; esta se entiende como la orientación de ciertas expresiones lingüísticas hacia un sistema de coordenadas presidido por el 'yo' (Vicente Mateu, 1994). En ese sistema de coordenadas participa, por ejemplo, la deixis; el mayor o menor grado de determinación deíctica presupone un nivel paralelo de egocentrismo (Rauh, 1983). Todo ello podría explicar el tradicional 'carácter egocéntrico' tradicionalmente atribuido al discurso oral (Beinhauer, 1968; Vigara Tauste, 1980; Padilla García, 2004; Briz, 2010).

Sin embargo, Vicente Mateu (1994) apunta la existencia de diferencias entre el egocentrismo y la subjetividad: el primero consiste en un principio organizador de las expresiones deícticas, tanto en el sistema como en el uso actualizado, mientras que la segunda la define como el principio de expresiones del sistema y como principal manifestación pragmática del sujeto que razona intencionadamente. Así, siguiendo al autor, el egocentrismo apunta a una determinada estructura lingüística, mientras que la subjetividad refleja un juicio, opinión o estado afectivo del hablante. Tanto los estudios sobre egocentrismo como los dedicados a la subjetividad lingüística están fundamentados en la perspectiva textual y pragmática. 'La inscripción de los interlocutores en el texto', la 'modalización' y la 'polifonía' son rasgos que han ido perfilando la segunda (Calsamiglia \& Tusón, 1999). Por otra parte, el uso de los pronombres de primera persona en distintas lenguas ha sido considerado como un modo de construcción y proyección de distintos aspectos sociales y de identidad personal (Englebretson, 2007). 
A pesar de estos aparentes avances, el enfoque cognitivo de la subjetividad/ objetividad como categorización estilística en el discurso no ha sido suficientemente explorado, lo cual ha dado lugar a que la subjetividad lingüística se conciba habitualmente como una gama de connotaciones textuales, se aplique de forma independiente al estudio de construcciones lingüísticas concretas, y se simplifique asociándolo a los textos de naturaleza oral, generalmente calificados como 'coloquiales' o 'informales'.

\subsection{Gramática y cognición en la variación entre la expresión y la omisión de 'yo'}

De acuerdo con la naturaleza icónica, simbólica y gradual dela gramática (Langacker, 1991, 2009; Croft \& Cruse, 2008), el enfoque cognitivo explica adecuadamente el funcionamiento de las variantes expresa (preverbal y posverbal) y omitida de los pronombres de sujeto ${ }^{4}$, ya que cada una de ellas implica una conceptualización distinta del referente: la omitida presupone que este es prominente, accesible o se encuentra activado en la enunciación ( $\varnothing$ Viene a las siete), mientras que la expresa implica hacer presente a dicho referente como centro sobre el cual se enmarca la predicación ('María' viene a las siete). Estos hechos básicos de la variación en la expresión del sujeto pueden conceptualizarse según las nociones de 'prominencia cognitiva' e 'informatividad textual', las cuales, concebidas idealmente como contrapuestas en los polos de una escala, permiten explicar la variación de los pronombres de sujeto (Serrano \& Aijón Oliva, 2011).

Mientras que la prominencia se centra en el grado de atención que recibe una unidad activada o accesible para el hablante, la informatividad se corresponde con el grado de novedad o de imprevisibilidad textual; lo menos esperable en un determinado contexto es lo más informativo. Según Beaugrande y Dressler (1997), la informatividad de un texto está basada en la focalización de la atención de los hablantes y oyentes. Así entendida, presupone un mayor esfuerzo de procesamiento, esto es, a mayor imprevisibilidad, improbabilidad o novedad habrá mayor informatividad. La expresión del sujeto en español no es obligatoria, y no solamente conlleva siempre un significado distinto al referente y a la cláusula donde se inserta, sino también diversos matices discursivo-pragmáticos que surgen de tal significado. La omisión del sujeto pronominal constituirá, pues, el reflejo de la mayor prominencia cognitiva de su referente, mientras que un sujeto expreso indicará un grado más elevado de informatividad textual.

Por otra parte, hay que tener en cuenta que también la posición que ocupe el sujeto en la cláusula se halla relacionada con factores discursivos y cognitivos; hemos comprobado que la posposición, a pesar de su infrecuencia, proporciona al enunciado un enfoque cognitivo distinto al de la colocación preverbal, básicamente relacionado con la (menor) transitividad, la disminución de la agentividad del sujeto con respecto 
a la posición preverbal, y la naturaleza del lexema verbal (Posio, 2012; Serrano, 2012; Aijón Oliva \& Serrano, 2012a; Serrano, 2013a; Serrano \& Aijón Oliva, 2013a).

El sujeto preverbal ocupa la posición lineal canónica SV, queda destacada su agentividad y es probable que ya haya sido mencionado en el discurso precedente, por lo que resulta más prominente que la variante pospuesta. Esta última, en cambio, adopta una colocación más informativa, entendida como novedosa o imprevista, aportando una focalización pragmática. Por ello, su prominencia cognitiva y su agentividad disminuyen ${ }^{5}$.

Así pues, disponemos de este modo las variantes preverbal y posverbal del sujeto expreso, de acuerdo con la informatividad textual y la prominencia cognitiva (Tabla 1). Según queda reflejado, la variante preverbal es más prominente pero menos informativa que la posverbal, que adquiere los rasgos +informativo y -prominente, de modo que esta alcanza el mayor grado de informatividad.

Tabla 1. Gradación de la prominencia cognitiva y la informatividad textual de la colocación preverbal y posverbal del sujeto pronominal 'yo'.

\begin{tabular}{|c|c|}
\hline $\begin{array}{c}\text { Sujeto preverbal } \\
\text { ('Yo'vengo) }\end{array}$ & $\begin{array}{c}\text { Sujeto posverbal } \\
\text { (Vengo 'yo') }\end{array}$ \\
\hline -informativo & + informativo \\
+prominente & -prominente \\
\hline
\end{tabular}

Además, el significado que resulta del uso de una u otra variante del sujeto pronominal en el discurso y en la interacción permite la creación de estilos lingüísticos que caracterizan determinadas situaciones comunicativas y grupos sociales (Aijón Oliva \& Serrano 2010; Serrano \& Aijón Oliva, 2011).

\section{Marco metodológico}

A partir de estas nociones cognitivas y discursivo-pragmáticas señaladas en el apartado anterior, analizaremos el pronombre 'yo' cuando aparece formulado en el discurso ylos distintos significados dela posición preverbaly posverbal respectivamente. Para ello se analizará esta variación en distintos géneros conversacionales y de los medios de comunicación del Corpus Conversacional del Español de Canarias. Este corpus consta de dos secciones: una compuesta por un conjunto de textos procedentes de conversaciones espontáneas con un total de 79.544 palabras, y otra que forman textos orales procedentes de grabaciones de programas de la radio y la televisión autonómica canaria, que consta de 171.258 palabras. Los primeros corresponden a la transliteración de un total de 994 minutos de conversación, mientras que los textos de los medios de comunicación constan de un conjunto de textos de distintos géneros (entrevistas, debates y programas de entretenimiento) transliterados de distintos canales 
autonómicos canarios (Radiotelevisión Canaria, Canal 7 del Atlántico y Mírame TV). Por lo que respecta a las conversaciones espontáneas, la técnica para la obtención de los datos fue la de 'participante-observador', ya que el objetivo era obtener la forma de hablar lo más espontánea posible, dentro de las naturales restricciones que impone la 'paradoja del observador' (Labov, 1983). Para ello, no se utilizaron cuestionarios, entrevistas o formularios predeterminados, simplemente se grabaron conversaciones espontáneas de grupos de individuos en distintas situaciones comunicativas cotidianas y/o sociales: charlas entre amigos, compras, clases particulares, etc.

Los porcentajes han sido calculados con el paquete estadístico GoldVarbX (Sankoff, Tagliamonte \& Smith, 2005), que permite calcular los porcentajes de aparición de una variante y la tabulación cruzada de varios de ellos; asimismo analiza estadísticamente las probabilidades de aparición de una variante en relación a distintos factores.

Además, para complementar el análisis de frecuencias, en este trabajo utilizamos la metodología de la 'variable absoluta'. Este método permite comprobar la frecuencia de una variante en relación con otros elementos contextuales, considerando la forma por sí misma y no como alternativa a otra(s). Se realiza un cálculo de esa forma por cada 10.000 palabras y se divide por el total de palabras de otra categoría (lingüística o social) del corpus (Aijón Oliva \& Serrano, 2012b).

\section{Resultados. Distribución del uso y de la variación entre la expresión/omisión del pronombre 'yo' en los géneros textuales}

A continuación se presentan los resultados cualitativos y cuantitativos del análisis.

a) Frecuencia de elección de cada pronombre. Sin duda, el pronombre 'yo' es una forma muy frecuente en todos los géneros del corpus, y ello es debido a que se corresponde con la inevitable participación de la persona que habla en el discurso, pero es previsible que esta inserción difiera notablemente según las características del género de que se trate, y aún más importante, de la distribución en esos textos de las variantes expresa (preverbal o posverbal) y omitida.

Así pues, en los textos conversacionales hemos podido comprobar que es el pronombre más utilizado. De 1474 casos de sujeto, el 55,7\% corresponden a 'yo', datos que se refrendan con las frecuencias absolutas; las diferencias cuantitativas con el resto de los pronombres es ampliamente superior, e indica que en las conversaciones espontáneas hay una mayor participación de la primera y la segunda persona del singular, como cabía esperar (Tabla 2). En esta tabla se presenta una distribución de las frecuencias de todos los pronombres según los porcentajes y las frecuencias absolutas; en ambos casos, los resultados indican el predominio de la primera persona del singular. 
Tabla 2. Porcentajes y frecuencias absolutas de los pronombres de sujeto en los textos conversacionales del CCEC

\begin{tabular}{|l|c|c|}
\hline & Casos y porcentajes & Frecuencias absolutas \\
\hline Yo & $820(55,7 \%)$ & 103 \\
\hline Tú & $350(23,7 \%)$ & 44 \\
\hline Nosotros & $246(16,7 \%)$ & 30.9 \\
\hline Usted/ustedes & $58(3,9 \%)$ & 7.2 \\
\hline Total & $\mathbf{1 . 4 7 4}$ & \\
\hline
\end{tabular}

En los textos de los medios de comunicación del corpus, este pronombre obtiene también una frecuencia muy elevada, aunque en este caso ligeramente inferior a 'nosotros', y esto es debido a que en estos textos, este pronombre es el más frecuente, como se puede comprobar en la siguiente tabla (Tabla 3), donde queda claro que la primera persona del plural es más frecuente que la del singular en este tipo de texto.

Tabla 3. Porcentajes y frecuencias absolutas de los pronombres de sujeto en los textos de los medios de comunicación del CCEC.

\begin{tabular}{|l|c|c|}
\hline & Casos y porcentajes & Frecuencias absolutas \\
\hline Yo & $1180(35,6 \%)$ & 68.9 \\
\hline Tú & $227(6,9 \%)$ & 13.2 \\
\hline Nosotros & $1512(45,7 \%)$ & 88.2 \\
\hline Usted/ustedes & $392(11,8 \%)$ & 22.8 \\
\hline Total & 3311 & \\
\hline
\end{tabular}

b) Frecuencias de omisión y expresión. Por otra parte, en las dos clases de textos del corpus, la variante omitida de todos los pronombres es la predominante, a excepción de 'usted-ustedes' que, como ya hemos advertido anteriormente, se aparta de esta tendencia y aparece con mayor frecuencia expreso (Aijón Oliva, 2010; Serrano, 2012; Serrano, 2013b). Esto podría ser un primer indicio de que los textos conversacionales no son tan subjetivos o egocéntricos como se ha venido señalando. En las interacciones conversacionales, los participantes de primera y segunda persona están presentes, y dichas interacciones se desarrollan generalmente en torno a la modalidad dialogal, con la participación de varias personas que interactúan entre sí, de modo que no es necesario hacer uso de los pronombres, salvo que exista alguna necesidad discursivopragmática especial. Por otro lado, en las conversaciones espontáneas pueden concurrir otras funciones o tipos textuales: narración, argumentación, descripción, etc., que en géneros más especificados en cuanto a sus rasgos formales y funciones comunicativas. De hecho, la conversación espontánea ha sido denominada como 'protogénero', del cual surge el resto de las modalidades enunciativas y actúa como marco de las mismas, ya que, como señalan Calsamiglia y Tusón (1999: 32): "en una conversación se argumenta y se polemiza, se cuenta y se relata, se explica o se expone y se describe". 
En los textos de los medios, la mayor incidencia de la omisión sería explicable, además de por la prominencia de los referentes que caracteriza a algunos géneros (sobre todo a los menos argumentativos), por algunas funciones pragmáticas específicas comunicativamente necesarias: cortesía, deseo de generalización de los referentes, inclusión/exclusión del hablante y/o del interlocutor, etc., que vienen determinadas por la naturaleza de estos géneros, caracterizados por el uso de ciertas pautas comunicativas concretas, concretadas en el uso de ciertas construcciones lingüísticas.

Por otra parte, al cuantificar las variantes de cada pronombre, observamos que las frecuencias de 'yo' expreso en los segundos textos son superiores a las de los textos conversacionales, lo cual indica nuevamente que no es posible afirmar que sea siempre en estos donde se encuentra el uso más abundante de este pronombre, ni siquiera en su variante expresa. En la Tabla 4, puede comprobarse la alta frecuencia del pronombre 'yo' expreso en los textos de los medios de comunicación, cuyo porcentaje supera al de los conversacionales (55,4\% frente a 46,6\%).

Tabla 4. Porcentajes de la expresión y de la omisión de los pronombres de sujeto en los textos conversacionales y de los medios de comunicación del CCEC.

\begin{tabular}{|l|c|c|c|c|}
\hline & \multicolumn{2}{|c|}{ Textos conversacionales } & \multicolumn{2}{c|}{ Textos de los medios de comunicación } \\
\hline & Expresión & Omisión & Expresión & Omisión \\
\hline Yo & $382(46,6 \%)$ & $438(53,4 \%)$ & $654(55,4 \%)$ & $526(44,6 \%)$ \\
\hline Tú & $130(37,1 \%)$ & $220(62,9 \%)$ & $70(30,8 \%)$ & $157(69,2 \%)$ \\
\hline Nosotros & $41(16,7 \%)$ & $205(83,3 \%)$ & $117(7,7 \%)$ & $1395(92,3 \%)$ \\
\hline Usted-ustedes & $36(62 \%)$ & $22(38 \%)$ & $211(53,8 \%)$ & $181(46,2 \%)$ \\
\hline Total & $\mathbf{5 8 9}$ & $\mathbf{8 8 5}$ & $\mathbf{1 . 0 5 2}$ & $\mathbf{2 . 2 5 9}$ \\
\hline
\end{tabular}

El siguiente gráfico permite la comparación de todas las variantes de los pronombres en los dos subtipos de textos del corpus. La variante omitida de 'nosotros' omitida es la que alcanza una frecuencia superior con respecto a todo el conjunto del paradigma tanto en los textos conversacionales como en los de los medios de comunicación. El pronombre 'usted/ustedes' es el que obtiene un mayor porcentaje de frecuencias en ambos textos, ligeramente superior en los conversacionales. Por su parte, el pronombre 'yo' resulta estar un poco más equilibrado que el resto en cuanto a la expresión y omisión en los distintos tipos de texto, si bien, como se ha señalado, destaca el incremento de la expresión en los textos de los medios. 


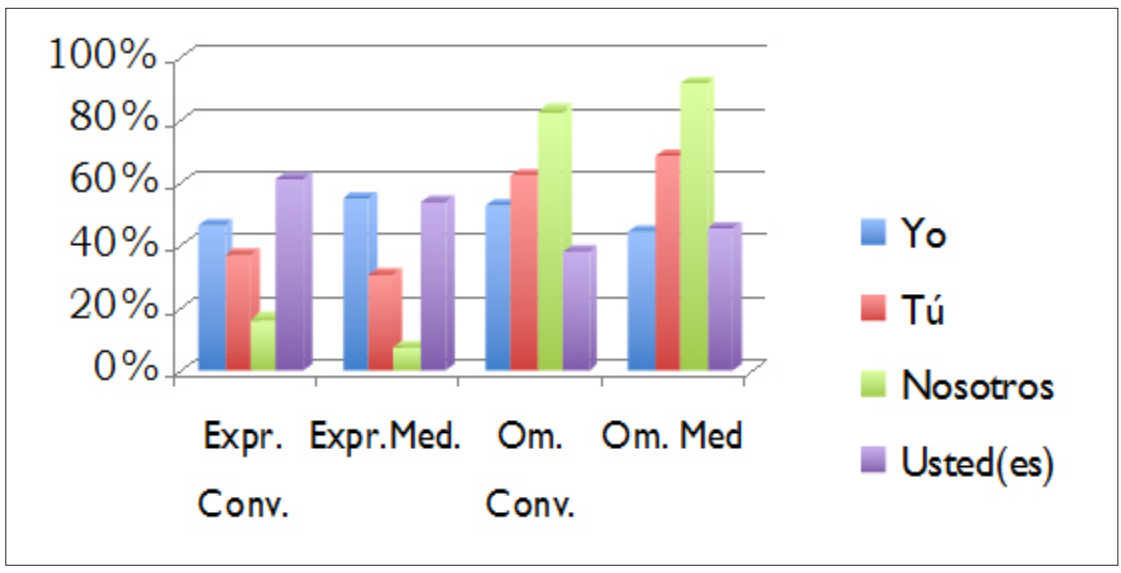

Gráfico 1. Comparación entre la expresión y omisión de los pronombres de sujeto en los dos tipos de textos del CCEC.

\section{Resultados. Variación situacionalydiscursivo-pragmática de la expresión (preverbal o posverbal) del pronombre ‘yo'}

\subsection{Variación situacional}

El significado del sujeto 'yo' expreso se concreta, además de por la gradación de las propiedades cognitivas señaladas anteriormente, por su aparición en determinadas situaciones comunicativas. A ello hay que añadir la observación de las variantes preverbal y posverbal, que, como hemos señalado, conllevan significados diferentes. Por tanto, su distinta frecuencia en los géneros de los medios de comunicación y en los conversacionales debe ser explicada de acuerdo a la posición que toma con respecto al verbo y a las características de los textos donde es más frecuente.

Como se describió anteriormente, en el plano comunicativo las variantes del sujeto expreso (preverbal y posverbal) aportan distintas perspectivas de la prominencia y la informatividad. Como se puede observar en los ejemplos siguientes (1) y (2), 'yo' expreso conlleva cierta prominencia; ha sido previamente mencionado, de modo que está activado o es accesible, pero no deja de mostrar la correspondiente informatividad textual, ya que su referente se presenta focalizado y realzado pragmáticamente, mostrando claramente su papel de agente de la acción verbal.

(1) 'Yo' no quiero decir que haya que tomar otras decisiones \pero las que se han tomado no son totalmente satisfactorias \hay que renovar los debates $\backslash$ $($ CCEC $<\operatorname{Med} 12>)$

(2) 'Yo' para los papeles soy un desastre y cuando 'yo' leí el papel $\backslash$ lo leí anoche y digo mi madre \qué es esto (CCEC $<$ ConvElEn>) 
En (1), mediante el uso del pronombre, el hablante muestra su posición con respecto al tema tratado, frente a otras posibles posturas que se han expuesto en la conversación. Destaca su papel de agente en esa opinión expresada, al concentrar la atención informativa sobre su propia persona. En (2), ambos usos de 'yo' antepuesto designan el protagonismo del hablante sobre el enunciado, de modo que queda destacada su agentividad con respecto a otras posibles personas del discurso.

Por el contrario, en la posición posverbal, al aparecer situado en la posición sintáctica típica de los objetos, hace disminuir claramente su agentividad (Verhagen, 1995) y se muestra como destino del evento designado por el lexema verbal.

(3) Digo 'yo' que lo que más ha molestado es lo de las prospecciones petrolíferas $\backslash$ más que la reducción de márgenes $\backslash$ porque en realidad ha sido una reducción de gastos $($ CCEC $<$ Med12 $>)$

(4) Tengo 'yo' unas fotos ahí \con corbataldonde se me ve con corbata $(\mathrm{CCEC}<\mathrm{ConvElEn08>)}$

Como se observa en los ejemplos anteriores (3) y (4), el pronombre no se presenta como actor del enunciado, sino como responsable o receptor del contenido del lexema verbal, con lo cual, adquiere cierta objetualización; el sujeto deja de ser agente y se implica como parte final del proceso de predicación, lo cual hace que el referente del sujeto se perciba como imprevisto, novedoso o focal, en virtud de la posición menos prototípica bajo la cual se presenta.

Por lo tanto, la variante posverbal incrementa la focalización y el realce pragmático consecuente de la informatividad (que también posee la variante preverbal) pero orienta el significado hacia la objetualización. Esto da lugar a que cada colocación enfoque la subjetividad propia del sujeto expreso de modo gradual (Serrano, 2012). En el siguiente gráfico se esquematizan las propiedades de la colocación preverbal o posverbal del sujeto pronominal (Cuadro 1). El sujeto preverbal es agentivo y adquiere los rasgos +subjetivo y -objetivo; el posverbal en cambio es objetual y focalizador, al tiempo que resulta +objetivo y-subjetivo.

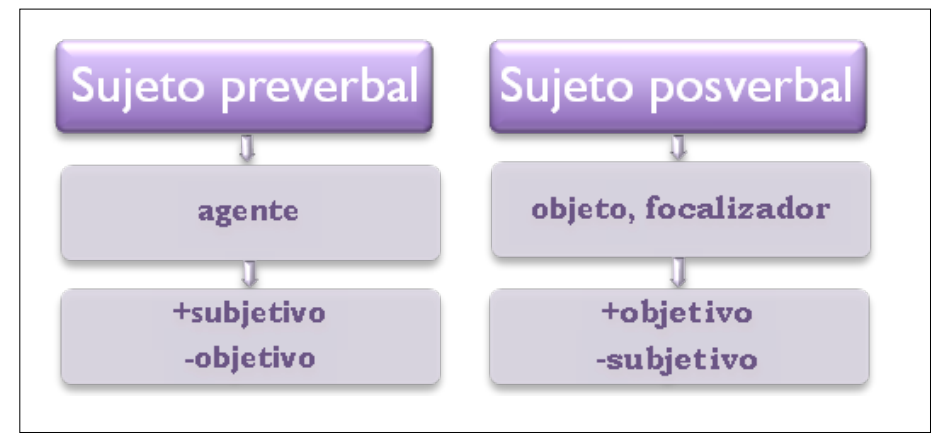

Cuadro 1. Valores cognitivos de la expresión preverbal y posverbal del sujeto pronominal. 
La distribución de las variantes preverbal y posverbal de 'yo' se manifiesta de forma diversa en los dos grupos de textos del corpus. La posverbal sigue siendo la menos frecuente, pero es considerablemente menor en los segundos (Tabla 5), con un escaso porcentaje de 3,7\% frente al 17,6\% que se obtiene en los textos conversacionales. En cambio, la frecuencia de la variante preverbal es muy elevada $(96,3 \%)$.

Tabla 5. Porcentajes de la colocación preverbal y posverbal en los textos conversacionales y de los medios de comunicación del CCEC.

\begin{tabular}{|l|c|c|c|}
\hline & Yo preverbal & Yo posverbal & Total \\
\hline Textos conversacionales & $315(82,4 \%)$ & $67(17,6 \%)$ & 382 \\
\hline Textos de los medios de comunicación & $630(96,3 \%)$ & $24(3,7 \%)$ & 654 \\
\hline
\end{tabular}

Para obtener datos más precisos y conocer con detalle la incidencia de cada variante, hemos calculado los porcentajes y las frecuencias absolutas de los distintos géneros de los medios de comunicación que contiene esta sección del corpus (Tabla 6). Estos cubren las principales modalidades discursivas de la televisión y la radio: a) magazines: programas de entretenimiento o variedades, en los que, gracias a su naturaleza diversa es posible encontrar distintas modalidades discursivas; b) informativos: programas dedicados estrictamente a la información donde a su vez aparecen secuencias de otros subtipos informativos: deportivos, meteorológicos, sociedad, etc.; c) tertulias: reuniones de periodistas y políticos (entre otros hablantes) que tratan sobre un tema de actualidad; d) informativos-debates: programas dedicados a la información sobre temas políticos y de actualidad con la presencia de diversos hablantes que confrontan sus opiniones.

Tabla 6. Porcentajes y frecuencias absolutas de la colocación preverbal y posverbal del pronombre 'yo' en los distintos géneros de los medios de comunicación del CCEC.

\begin{tabular}{|l|c|c|c|c|}
\hline & \multicolumn{2}{|c|}{ Yo preverbal } & \multicolumn{2}{c|}{ Yo posverbal } \\
\hline & Casos y porcentajes & Frecuencias Absolutas & Casos y porcentajes & Frecuencias absolutas \\
\hline $\begin{array}{l}\text { Conversaciones } \\
(79.544 \text { palabras) }\end{array}$ & $315(82,4 \%)$ & 39.6 & $67(17,6 \%)$ & 8.4 \\
\hline $\begin{array}{l}\text { Magazines } \\
(61.346 \text { palabras) }\end{array}$ & $160(95,2 \%)$ & 26 & $8(4,8 \%)$ & 0.13 \\
\hline $\begin{array}{l}\text { Informativo-debate } \\
(41.753 \text { palabras) }\end{array}$ & $283(98,3 \%)$ & 67.7 & $5(1,7 \%)$ & 0.11 \\
\hline $\begin{array}{l}\text { Tertulias } \\
(12.685 \text { palabras) }\end{array}$ & $75(97,5 \%)$ & 59.1 & $2(2,5 \%)$ & 0.15 \\
\hline $\begin{array}{l}\text { Informativos } \\
(55.474 \text { palabras })\end{array}$ & $112(92,6 \%)$ & 20.1 & $9(7,4 \%)$ & 0.16 \\
\hline
\end{tabular}

Tanto en los porcentajes como en las frecuencias absolutas, queda reflejado que la posición preverbal de 'yo' coaparece con aquellos textos más relacionados con la modalidad discursiva argumentativa (informativos-debates y tertulias), en las cuales el hablante establece su opinión o punto de vista a partir de la representación de su propia persona. Hay que destacar la elevada frecuencia del sujeto en los programas 
estrictamente informativos. Esto es debido a que en estas emisiones se producen frecuentes intervenciones de personas ajenas al programa que dan su opinión o parecer sobre alguna cuestión de actualidad. Aun así, las frecuencias absolutas muestran que en general la presencia de sujetos de primera persona es mucho menor que en otros géneros.

Este sujeto suele ser prominente, de modo que su formulación reafirma la presencia del hablante en el enunciado que emite, dotándolo cognitivamente de cierta subjetividad, como se observa en los ejemplos (5) y (6).

(Adán Martín) fue elegidoly 'yo' le fui a hacer una entrevistaly le dije Adán 'yo' te tengo que preguntar por la enfermedad $\backslash$ prefiero hacerlo contigo que sé que vas a reflejar la realidad $\backslash(\mathrm{CCEC}<\mathrm{Med} 12>)$

(6) 'Yo' he pasado momentos más alegres que tristes en la política\pero 'yo' he disfrutado mucho en la políticaly he disfrutado mucho en la vida $($ CCEC $<$ Med12>)

No obstante, estos resultados son hasta cierto punto previsibles; se presupone que un discurso argumentativo necesita formalizarse a través de determinados elementos lingüísticos, y la expresión preverbal del sujeto pronominal es uno de los más adecuados, ya que permite incrementar la agentividad del referente sobre el contenido proposicional, sin dejar de presentar, como hemos señalado, cierta prominencia. En los textos mencionados el hablante necesita hacer uso del pronombre 'yo' para afirmar su participación en el contenido que emite, señalando que se trata de su propia opinión, vivencia o argumento. Nótese que en el siguiente ejemplo (7) la presencia del pronombre 'yo' da lugar a este significado argumentativo o de opinión.

(7) 'Yo' no pretendo llegar a la mayoría absoluta\'yo' no digo que vayamos a tener la mayoría absoluta \pero sí digo que ahora mismo este partido es el partido de la mayoría absoluta $\backslash(\mathrm{CCEC}<\operatorname{Med} 12>)$

En cambio, resulta llamativo que las frecuencias sean bastante inferiores en los textos conversacionales, a los que usualmente se les atribuye una mayor subjetividad, (normalmente se les adjudica una mayor 'coloquialidad', 'informalidad' o 'egocentrismo', términos que en general no han sido definidos y aplicados con el suficiente rigor), y que se hallen incluso por debajo de las de los géneros puramente informativos. En las conversaciones, las necesidades argumentativas de los participantes disminuyen porque generalmente la diversidad de temas tratados es más amplia que en las tertulias y debates y, como se señaló anteriormente, estos textos no tienen una finalidad comunicativa concreta, como sí la suelen tener los textos de los medios. Asimismo, los participantes suelen estar presentes en la interacción, de modo que también decrece la necesidad de indexar al interlocutor mediante el uso de los pronombres, como se observa en los ejemplos (8) y (9). 
(8) También sé que $\varnothing$ tienen presupuesto para la cocina desde hace meses así $\backslash$ que no sé \¿sospechas?/ (CCEC<ConvElEn08>)

(9) Lo primero que $\varnothing$ tienes que hacer $\backslash$ es lo que está multiplicando y dividiendo hacerlo parejas $\backslash(\mathrm{CCEC}<$ ConvElEn08 $>)$

Al mismo tiempo, la posición posverbal presenta unas frecuencias muy bajas en los textos de los medios de comunicación, pero no en los conversacionales $(17,6 \%$ y $8,4 \%$ de frecuencia absoluta), lo cual indica que esta variante puede ser considerada como más característica de los textos conversacionales que de aquellos de los medios de comunicación (ejemplos 10 y 11).

(10) A: ¿Este problema lo hiciste tú solita?

B: Sí \señorita \inicialmente lo hice 'yo’ \y en otra hoja lo escribo 'yo' de nuevo (CCEC <ElEn08>)

(11) El año pasado estaba mala, casi no podía ni comer ni nada||pero no fui porque no quería 'yo' ir III y un año la (la Navidad) pasé 'yo' en la Residencia (CCEC $<$ ElEn08>)

Este ligero incremento de la variante posverbal en los textos conversacionales, y su consecuente significado [-subjetivo] que hemos descrito, reafirmaría la idea de que la subjetividad es mayor en los textos de los medios de comunicación que en los conversacionales.

Esto puede confirmar la idea que hemos venido apuntando; la conversación espontánea no debería ser considerada como el texto de mayor representación de la subjetividad ${ }^{6}$. Las respectivas frecuencias de la colocación del pronombre expreso 'yo', indican que la naturaleza de estos textos no propicia el uso del sujeto expreso, bien sea porque los referentes están plenamente accesibles en el transcurso de la interacción, bien sea porque las distintas modalidades enunciativas de las conversaciones diversifican la necesidad de la representación del referente a través del pronombre. Esto no promueve el uso expreso de los pronombres, al contrario de lo que sucede en otros géneros de los medios, como los debates, donde la dinámica interactiva de defensa de posturas y opiniones personales provoca el uso expreso (sobre todo en posición preverbal) de las formas pronominales. De hecho, Scheibman (2007) llega a una conclusión similar en un estudio sobre las conversaciones espontáneas del inglés, donde se obtuvo una proporción muy alta de construcciones y referencias inespecíficas o generalizadoras. Asimismo, Haddington (2007) encuentra que ciertas estructuras y construcciones dotadas de subjetividad aparecen en los textos de los medios de comunicación de modo más sistemático que en las conversaciones espontáneas.

De este modo, podemos caracterizar como de menor a mayor subjetividad los distintos géneros analizados, según se muestra en el siguiente gráfico. La curva 
representa el incremento del grado de subjetividad de los distintos textos del corpus; la menor subjetividad la obtendrían los textos conversacionales y la mayor los informativos-debates. Después de las conversaciones, los niveles inferiores de subjetividad los obtienen, como sería esperable, los informativos, y aumenta progresivamente en los magazines y en las tertulias. Todo ello debido a las razones argumentadas anteriormente.

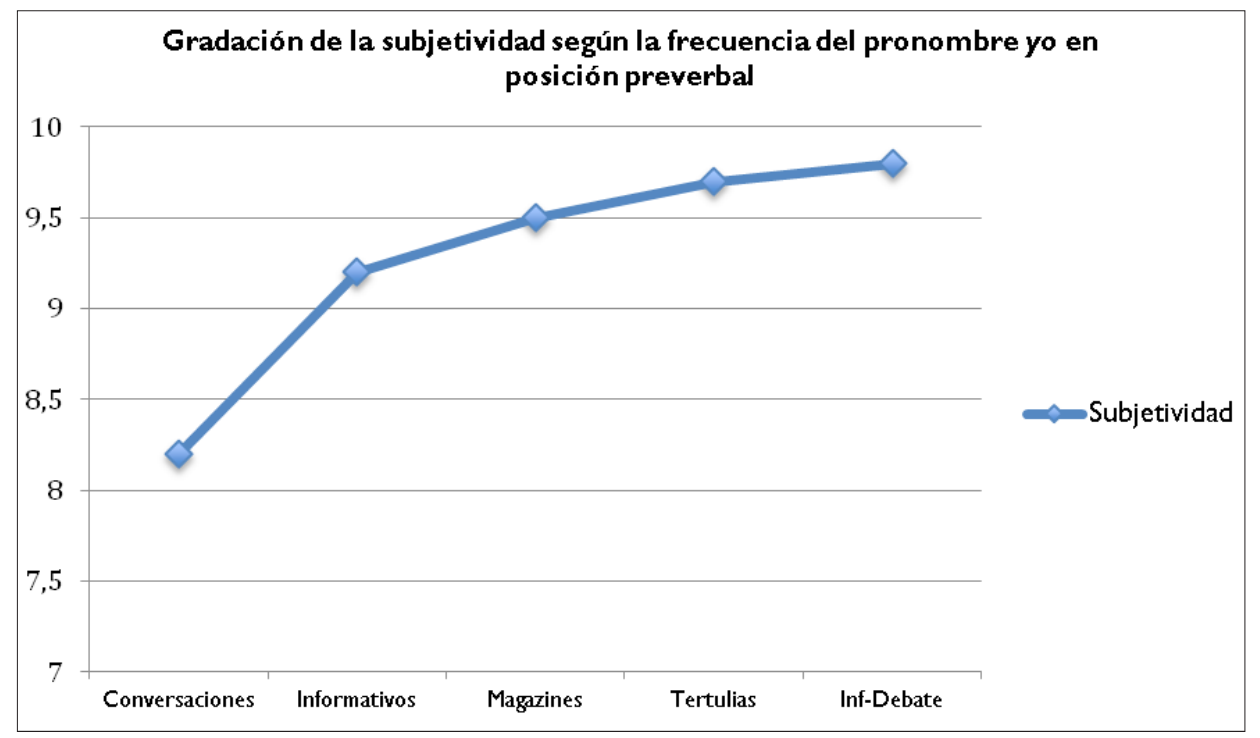

Gráfico 2. Gradación de la subjetividad en los distintos géneros textuales del CCEC.

\subsection{Variación discursivo-pragmática de 'yo': Los lexemas verbales}

La hipótesis de que la expresión del sujeto de primera persona es un medio de subjetivación del discurso (Aijón Oliva \& Serrano, 2010; Aijón Oliva, 2012) adquiere más relevancia si se relaciona con los lexemas verbales con los que aparece. De hecho, en estudios precedentes, centrados en tipos de verbos concretos, como 'creer' (Aijón Oliva \& Serrano, 2010) o 'decir' (Aijón Oliva, 2012), hemos concluido que tanto uno como otro obtienen una alta frecuencia en los textos de los medios de comunicación. En este trabajo se puede confirmar que, de los 654 casos de 'yo' expreso, 345 vienen formulados con uno de estos dos verbos, lo cual constituye un 52,7 \% del total de los casos de 'yo'. En la Tabla 7 se presenta la distribución de las posiciones preverbal y posverbal de este pronombre en los textos de los medios de comunicación. Como claramente se observa, la posición posverbal es muy escasa en ambos verbos y, aunque los casos del lexema 'decir' no son muy elevados, siguen la misma tendencia de los de 'creer'. 
Tabla 7. Casos y porcentajes de la expresión del pronombre ‘yo' preverbal y posverbal con los verbos 'creer' y ‘decir' en los textos de los medios de comunicación del CCEC.

\begin{tabular}{|l|c|c|c|}
\hline & Preverbal & Posverbal & Total \\
\hline Creer & 301 & 6 & 307 \\
\hline Decir & 25 & 13 & 38 \\
\hline & & & 345 \\
\hline
\end{tabular}

En los siguientes ejemplos (12 y 13) se pueden advertir estos usos.

(12) 'Yo creo' que hay un dato importante \yo aquí quiero dejar claro que la confianza en el gobierno|| 'yo creo' que la imagen de este gobierno es bastante positiva\ $($ CCEC $<$ Med12>)

(13) Pero 'yo digo' que en 2011 nos hemos pasado el año|||el año de elecciones $\backslash$ pasamos a una crisis que afecta a todos los países desarrollados $\backslash(\mathrm{CCEC}<\mathrm{Med12}>)$

En cambio, en los textos conversacionales, la frecuencia de estos lexemas verbales es mucho menor; solo un 9,4\% de los 382 sujetos se expresa con alguno de ellos. En la tabla 8 queda expuesta la distribución de cada variante con los dos lexemas verbales.

Tabla 8. Casos y porcentajes de la expresión del pronombre ‘yo' preverbal y posverbal con los verbos 'creer' y 'decir' en los textos conversacionales del CCEC.

\begin{tabular}{|l|c|c|c|}
\hline & Preverbal & Posverbal & Total \\
\hline Creer & 18 & 2 & 20 \\
\hline Decir & 12 & 4 & 16 \\
\hline & & & 36 \\
\hline
\end{tabular}

Los casos indican que los significados cognitivos y discursivos de la expresión del sujeto tienden a coaparecer de modo más frecuente con verbos de dicendi e intelligendi, y que esta conjunción obtiene su máxima rentabilidad comunicativa en los textos de los medios de comunicación. El significado intrínseco de estos verbos permite al hablante conformar una posición o actitud personal que lo valida como responsable del contenido que emite, lo que resulta muy propicio para los textos en los que aparece. Esto sería un indicio más de que el sujeto expreso, y concretamente el preverbal, contribuye a la subjetivación del discurso, la cual se incrementa en aquellos géneros que, a su vez, están caracterizados por cierta tendencia a la enunciación de tipo argumentativo, como los debates, las tertulias y los magazines ${ }^{7}$. Las conversaciones espontáneas, por contra, muestran una tendencia a la subjetivación bastante inferior, como se deduce de las frecuencias. No obstante, se requiere un estudio cualitativo exhaustivo sobre la colocación del sujeto y la naturaleza del lexema verbal para confirmar esta idea.

\section{CONCLUSIONES}

Desde una perspectiva cognitiva como la que aquí adoptamos, la subjetividad es el producto de la conceptualización de la realidad desde el punto de vista de los sujetos 
y, consecuentemente, desplazada de los objetos (Cuenca \& Hilferty, 1999; Croft \& Cruse, 2008), o bien la manifestación del punto de vista o actitud del hablante hacia el discurso o el contenido proposicional (Scheibman, 2007). La dimensión gradual de la 'objetividad-subjetividad', por tanto, se entiende como una consecuencia del uso de las distintas variantes del sujeto pronominal y el significado cognitivo que aportan cada una de ellas. Cuando la gramática ofrece distintas posibilidades de describir la realidad, la elección del hablante puede ser indicativa de un enfoque orientado cognitivamente hacia la subjetividad (Israeli, 1997; Scheibman, 2001), lo cual, al mismo tiempo, muestra la evidencia de que las estructuras gramaticales sirven a distintos propósitos comunicativos en el discurso, como es el caso del uso de la primera persona del paradigma pronominal (Englebretson, 2007).

La inscripción del referente en el enunciado a través del uso del sujeto expreso en conjunción con la naturaleza discursiva de los géneros con los que aparece, y con otros elementos discursivo-pragmáticos como el lexema verbal, hace posible que la comunicación se incline hacia el polo de la subjetividad. Esta subjetividad se matiza con la distinta perspectiva que de ella ofrecen respectivamente la posición preverbal y posverbal del sujeto pronominal; la primera lo hará con mayor intensidad que la segunda. Se trata, por tanto, de una subjetividad fundamentada en la percepción cognitiva de la persona y su referente en la interacción comunicativa, lo cual consiste en una perspectiva diferente a la subjetividad que tradicionalmente se le atribuye a la lengua (sobre todo a la oral). Las conversaciones estarían caracterizadas por la 'intersubjetividad', o el conjunto de normas y conocimientos compartidos que orienta a los hablantes al uso de determinadas construcciones lingüísticas en una interacción comunicativa concreta (Scheibman, 2007), si bien está presente también en otro tipo de textos (Haddington, 2007).

De hecho, cualquier práctica socio-discursiva refleja el punto de vista subjetivo que ejerce el hablante sobre el enunciado que emite (Langacker, 1993); pero esta subjetividad será gradual y estará moldeada y concretada a través del significado que proporcionen las distintas formas y construcciones lingüísticas.

El significado que conlleva la variación sintáctica, observado en los distintos géneros textuales donde aparece, y otras características discursivas permiten determinar su contribución a la creación de estilos comunicativos que se vuelven regulares en ciertas situaciones comunicativas. En este caso, la expresión del sujeto preverbal parece característica de aquellos géneros de los medios de comunicación orientados comunicativamente hacia la argumentación o la expresión de ideas y opiniones personales, constituyendo así un rasgo o elemento que aporta subjetividad al discurso.

Además de su frecuencia en este tipo de textos, otro análisis deberá determinar su distribución social; es previsible que los participantes de estos géneros hagan un uso desigual de estas variantes, lo cual contribuirá a explicar de forma más precisa el significado de las mismas. 


\section{REFERENCIAS BIBLIOGRÁFICAS}

Aijón Oliva, M. A. (2010). Usted ya sabe lo que ya sabe usted: Aspectos discursivo-cognitivos de la colocación del sujeto pronominal. Ponencia presentada en el IX Congreso de Lingüística General, Universidad de Valladolid, España.

Aijón Oliva, M. A. (2012). Variación formal y proyección discursiva del sujeto yo con verba dicendi. Ponencia presentada en el X Congreso Internacional de Lingüística General, Universidad de Zaragoza, España.

Aijón Oliva, M. A. \& Serrano, M. J. (2010). El hablante en su discurso: Expresión y omisión del sujeto de creo. Oralia, 13, 7-38.

Aijón Oliva, M. A. \& Serrano, M. J. (2012a). La posición del sujeto pronominal en las cláusulas no declarativas. Onomázein, 26(2), 131-164.

Aijón Oliva, M. A. \& Serrano, M. J. (2012b). Towards a comprehensive view of variation in language: The absolute variable. Language \& Communication, 32, 80-94.

Atkins, K. (2005). Self and subjectivity. Oxford: Blackwell.

Beaugrande, R. \& Dressler, W. U (1997). Introducción a la Lingüistica del texto. Barcelona: Ariel.

Benveniste, E. (1977). Problemas de Lingüistica General II. México: Siglo Veintiuno Editores.

Beinhauer, W. (1968). El español coloquial. Madrid: Gredos.

Biber, D. \& Conrad, S. (2009). Register, genre, and style. Cambridge: Cambridge University Press.

Briz, A. (2010). Comentario de textos coloquiales. Madrid: Arco Libros.

Calsamiglia, H. \& Tusón, A. (2007). Las cosas del decir. Manual de Análisis del Discurso. Barcelona: Ariel.

Criado de Val, M. (1973). Gramática española y comentario de textos. Madrid: SAETA.

Croft, W. \& Cruse, D. A. (2008). Lingüística cognitiva. Madrid: Akal Cambridge.

Chen, W. (2008). Dimensions of subjectivity in natural languages. Ponencia presentada en el 46th Annual Meeting of the Association for Computational Linguistics on Human Language Technologies, 13-16.

Cuenca, M. J. \& Hilferty, J. (1999). Introducción a la Lingüistica Cognitiva. Barcelona: Ariel. 
Dorgeloh, H. \& Wanner, A. (2010). (Eds.) Syntactic variation and genre. Berlín: Mouton de Gruyter.

Ejarque, D. (1977). El pronombre personal sujeto en español. Cuadernos de Filología, 7, 29-84.

Englebretson, R. (2007). Stancetaking in discourse: Subjectivity, interaction, evaluation. Ámsterdam: John Benjamins.

Enríquez, E. (1984). El pronombre personal sujeto en la lengua española hablada en Madrid. Madrid: CSIC.

Fernández Ramírez, S. (1987). Gramática española. El pronombre. Madrid: Arco Libros.

Finegan, E. (1995). Subjectivity and subjectivisation: An introduction. En S. Dieter \& S. Wright (Eds.), Subjectivity and Subjectivisation: Linguistic Perspectives (pp.1-15). Cambridge: Cambridge University Press.

Haddington, P. (2007). Stancetaking as an Interactional Activity: Challenging the prior speaker. En R. Englebretson (Ed.), Stancetaking in Discourse Subjectivity, Interaction, Evaluation (pp. 253-282). Ámsterdam: John Benjamins.

Hale, K. (1966). Kinship reflections in syntax: Some Australian languages. Word, 22, 318-324.

Hernando Cuadrado, L. A. (1988). El español coloquial en El Jarama. Madrid: Playor.

Hidalgo Navarro, A. \& Pérez Jiménez, M. (2004). De la sintaxis a la pragmasintaxis: Problemas del análisis sintáctico en el discurso oral espontáneo. Cauce, 27, 221-245.

Israeli, A. (1997). Semantics and pragmatics of the reflexive verbs in Russian. Slavistische Beiträge. München: Verlag Otto Sagner.

Jensen, J. B. (1973). The feature [human] as a constraint on the occurrence of third person subject pronouns in Spanish. Hispania, 56(1),116-122.

Kerbrat-Orecchioni, C. (1980). La enunciación. De la subjetividad en el lenguaje. Buenos Aires: Hachette.

Labov, W. (1983). Modelos sociolingüisticos. Madrid: Cátedra.

Langacker, R. W. (1991). Concept, image, and symbol: The cognitive basis of grammar. Berlín: Mouton de Gruyter.

Langacker, R.W. (1993). Reference-point constructions. Cognitive Linguistics, 4, 1-38. 
Langacker, R. W. (2000). Estructura de la cláusula en la gramática cognoscitiva. En R. Maldonado (Ed.), Estudios cognoscitivos del español (pp. 19-65). Volumen monográfico de la Revista Española de Lingüistica Aplicada.

Langacker, R. W. (2009). Investigations in cognitive grammar. Berlín: Mouton de Gruyter.

Lavandera, B. (1984). Variación y significado. Buenos Aires: Hachette.

Narbona, A. (1991). Sintaxis coloquial y análisis del discurso. Revista Española de Lingüistica, 2(2),187-204.

Padilla García, X. A. (2001). El orden de palabras en el español coloquial. Valencia: Universidad de Valencia.

Padilla García, X. A. (2004). Del oyente receptor al oyente combatiente. Estudios de Lingüistica de la Universidad de Alicante, 18, 213-230.

Posio, P. (2012). The functions of postverbal pronominal subjects in spoken Peninsular Spanish and European Portuguese. Studies in Hispanic and Lusophone Linguistics, 5(1), 149-190.

Rauh, G. (1983). Essays on deixis. Tübingen: Beiträge zur Linguistic.

Rosengren, P. (1974). Presencia y ausencia de los pronombres de sujeto en español moderno. Acta Universitatis Gothoburgensis. Estocolmo: Almqvist \& Wiksell.

Sankoff, D., Tagliamonte, S. \& Smith, E. (2005). GoldVarb X: A multivariate Analysis Application for Macintosh and Windows. Toronto: University of Toronto.

Scheibman, J. (2001). Local patterns of subjectivity in person and verb type in American English conversation. En J. Bybee \& P. Hopper (Eds.), Frequency and the Emergence of Linguistic Structure (pp.61-89). Ámsterdam: John Benjamins.

Scheibman, J. (2007). Subjective and intersubjective uses of generalizations in English conversation. En R. Englebretson (Ed.), Stancetaking in Discourse Subjectivity, Interaction, Evaluation (pp. 111-138). Ámsterdam: John Benjamins.

Serrano, M. J. (2011). Otras personas y yo. Variación socioestilística de la expresión/ omisión del sujeto pronominal nosotros en las conversaciones espontáneas. En M. J. Serrano (Ed.), Variación Variable (pp. 93-126). Almería: Círculo Rojo/Ministerio de Ciencia e Innovación.

Serrano, M. J. (2012). El sujeto pronominal usted/ustedes y su posición: Variación y creación de estilos comunicativos. Spanish in Context, 9(1), 109-131.

Serrano, M. J. (2013a). De la cognición al texto: La prominencia cognitiva y la informatividad discursiva en el estudio de la variación de los sujetos pronominales. Estudios de Lingüistica de la Universidad de Alicante (en prensa). 
Serrano, M. J. (2013b). Variación sociosituacional de la colocación del sujeto pronominal en textos conversacionales. Spanish in Context, 10(2), 261-283.

Serrano, M. J. \& Aijón Oliva, M. A. (2010). La posición variable del sujeto pronominal en relación con la cortesía interactiva. Pragmaligüística, 18, 170-204.

Serrano, M. J. \& Aijón Oliva, M. A. (2011). Syntactic variation and communicative style. Language Sciences, 33, 138-153.

Serrano, M. J. \& Aijón Oliva, M. A. (2012). Cuando tú eres yo: La inespecificidad referencial de tú como recurso de objetivación en el discurso. Nueva Revista de Filología Hispánica (en prensa).

Serrano, M. J. \& Aijón Oliva, M. A. (2013a). El sujeto posverbal: Función pragmática y cognición en las cláusulas declarativas. Neuphilologische Mitteilungen (en prensa).

Serrano, M. J. \& Aijón Oliva, M. A. (2013b). Discourse objectivization, Social variation and style in the use of Spanish tú. Folia Linguistica, 47 (en prensa).

Verhagen, A. (1995). Subjectification, syntax, and communication. En S. Dieter \& S. Wright (Eds.), Subjectivity and Subjectivisation: Linguistic Perspectives (pp.103-128). Cambridge: Cambridge University Press.

Vicente Mateu, J. A. (1994). La deíxis. Egocentrismo y subjetividad en el lenguaje. Tesis de doctorado, Universidad de Murcia, España.

Vigara Tauste, A. M. (1980). Aspectos del español hablado. Madrid: SGEL.

Vigara Tauste, A. M. (2005). Morfosintaxis del español coloquial. Esbozo pragmaestilistico. Madrid: Gredos.

Wierzbicka, A. (1988). The semantics of grammar. Ámsterdam: John Benjamins.

\section{NOTAS}

1 Este trabajo forma parte del desarrollo del proyecto de I+D "Los estilos de comunicación y sus bases cognitivas en el estudio de la variación sintáctica en español" (FFI2009-07181/FILO).

2 No obstante, Jensen (1973) concluye que los sujetos de tercera persona pueden ser tan animados como los de primera y segunda, lo que podría conllevar la adopción de la misma prominencia que estos.

3 El autor señala que hay cualidades o sustantivos que pueden ser medidos objetivamente, como la temperatura o el tiempo, pero otros, como la belleza o la sabiduría no pueden ser medidos objetivamente, con lo cual, se encuentran siempre en planos subjetivos (2008:15).

4 Véase Aijón Oliva y Serrano (2010), Aijón Oliva (2012), para el pronombre 'yo'; Serrano y Aijón Oliva (2013b) para el pronombre 'tú'. Las formas 'usted/ustedes' se estudian en Aijón Oliva (2010) y Serrano (2012); y el pronombre 'nosotros' en Serrano (2011). 
5 Esto no implica que el sujeto posverbal no haya podido ser previamente mencionado en la interacción (Posio, 2012).

6 En otros trabajos basados en textos conversacionales, concretamente sobre el pronombre 'nosotros' (Serrano, 2011), hemos alcanzado la misma conclusión.

7 Sin embargo, no hay que olvidar que variación y texto o género se construyen mutuamente; que estos sean más subjetivos u orientados a la argumentación viene determinado por el uso de determinadas formas lingüísticas que contribuyan a ello, del mismo modo que las variantes adquieren buena parte de su significado a partir del texto o género donde se utilizan con mayor frecuencia. 